This is a self-archived version of an original article. This version may differ from the original in pagination and typographic details.

Author(s): Kostensalo, Joel; Suhonen, Jouni

Title: Beta-spectrum shapes of forbidden $\beta$ decays

Year: 2018

Version: Accepted version (Final draft)

Copyright: (c) World Scientific Publishing Company, 2018.

Rights: In Copyright

Rights url: http://rightsstatements.org/page//nC/1.0/?language=en

Please cite the original version:

Kostensalo, J., \& Suhonen, J. (2018). Beta-spectrum shapes of forbidden $\beta$ decays. International Journal of Modern Physics A, 33(9), Article 1843008.

https://doi.org/10.1142/S0217751X1843008X 


\title{
Beta-spectrum shapes of forbidden $\beta$ decays
}

\author{
Joel Kostensalo and Jouni Suhonen \\ Department of Physics, University of Jyvaskyla, P.O. Box 35 \\ Jyvaskyla, FI-40014, Finland \\ jouni.suhonen@phys.jyu.fi \\ Received Day Month Year \\ Revised Day Month Year
}

\begin{abstract}
The neutrinoless $\beta \beta$ decay of atomic nuclei continues to attract fervent interest due to its potential to confirm the possible Majorana nature of the neutrino, and thus the nonconservation of the lepton number. At the same time the direct dark-matter experiments are looking for WIMPs (weakly interacting massive particles) through their scattering on nuclei. The neutrino-oscillation experiments on reactor antineutrinos base their analyses on speculations of $\beta$-spectrum shapes of nuclear decays, thus leading to the notorious "reactor antineutrino anomaly". In all these experimental efforts one encounters the problem of $\beta$-spectrum shapes of forbidden $\beta$ decays, either as unwanted backgrounds or unknown components in the analyses of data. In this work the problem of spectrum shapes is discussed and illustrated with a set of selected examples. The relation of the $\beta$-spectrum shapes to the problem of the effective value of the weak axial-vector coupling strength $g_{\mathrm{A}}$ and the enhancement of the axial-charge matrix element is also pointed out.

Keywords: Forbidden beta decays; beta spectrum shapes; double beta decay; direct darkmatter search; reactor antineutrino anomaly; axial-vector coupling strength; axial-charge matrix element
\end{abstract}

PACS numbers: 21.60.Cs, 21.60.Jz, 23.40.Bw, 23.40.Hc

\section{Introduction}

At the moment the neutrinoless $\beta \beta(0 \nu \beta \beta)$ decay is the only practical means of accessing the possible Majorana nature of the neutrino. In order to proceed the decay requires the violation of lepton-number conservation and a non-zero neutrino mass. There are a host of different possible mechanisms which mediate the decay. ${ }^{1}$ The $0 \nu \beta \beta$ decay is related to nuclear structure via the nuclear matrix elements (NMEs), calculated in many different theory frameworks (see the reviews ${ }^{2-5}$ ), e.g. the quasiparticle random-phase approximation. ${ }^{6-8}$ Due to its great potential to revolutionize the field of electroweak interactions, the $0 \nu \beta \beta$ has attracted and continues to attract intense experimental interest. The presently running $0 \nu \beta \beta$ experiments include AURORA, GERDA, NEMO-3, COBRA, CUORE, EXO and KamLANDZen. $^{9-15}$ The future experiments include Majorana, SuperNEMO, MOON, AMoRE, LUMINEU, CUPID, SNO+, NEXT, and PandaX-III. ${ }^{16-24}$ Some of these secondorder processes compete with extremely retarded first-order processes, namely $\beta$ 
decays in the ${ }^{48} \mathrm{Ca}-{ }^{48} \mathrm{Sc}-{ }^{48} \mathrm{Ti}$ system ${ }^{25,26}$ and in the ${ }^{96} \mathrm{Zr}-{ }^{96} \mathrm{Nb}-{ }^{96} \mathrm{Mo}$ system. ${ }^{27,28}$ Many of the listed experiments are based on liquid scintillators which have light nuclei as cosmogenic backgrounds. Also very heavy nuclei like ${ }^{214} \mathrm{Bi}$ can be a dangerous background in $0 \nu \beta \beta$ experiments.

At the present there are many candidates for the WIMPs (weakly interacting massive particles). Probably the most popular one is the LSP (lightest supersymmetric particle). ${ }^{29-35}$ The WIMPs are expected to be non-relativistic and they can be detected by their scattering on atomic nuclei. There is a vast number of direct dark-matter detection experiments with different target materials: XENON10, XENON100, XMASS, ZEPLIN, PANDA-X, LUX, CDMS, CoGENT, EDELWEISS, DAMA, KIMS, PICASSO, ADMX, CDEX, CRESST, DarkSide, DMTPC, DRIFT and SIMPLE. ${ }^{36-54}$ Many of these experiments suffer from backgrounds produced by unknown $\beta$-spectrum shapes.

A further interesting application of the $\beta$-spectrum studies is the reactor antineutrino anomaly (RAA). ${ }^{55}$ The antineutrino spectra in the nuclear reactors result from the long uranium and plutonium $\alpha$ and $\beta^{-}$decay chains and the subsequent fission used as fuel to drive the energy production in the nuclear power plants. In the RAA the experimentaly measured antineutrino flux is lower than what is expected from the $\beta$ decays of the nuclear fission fragments deduced from nuclear data with some approximations. ${ }^{56}$ The method of virtual $\beta$ branches ${ }^{57-59}$ has been used to aid in the process of collecting the cumulative $\beta$ spectra responsible for the estimated theoretical antineutrino flux. The involved $\beta$ decays go partly by forbidden transitions that cannot be assessed by the present nuclear data, but instead, could be calculated by the formalism introduced in. ${ }^{60,61}$ The mesured flux is some $6(2) \%$ lower making this a rough $3 \sigma$ deviation. ${ }^{62}$ Possible shortcomings of the used analysis methods have been pointed out in. ${ }^{63}$

Beyond the half-life analyses (see, e.g. ${ }^{64-68}$ ) the $\beta$-spectrum shapes can also be used to pin down the effective value of the weak axial-vector coupling strength $g_{\mathrm{A}}$ in forbidden non-unique $\beta$ decays. In some $\beta$-decay transitions the shape of the $\beta$ spectrum is quite sensitive to the variations in the value of $g_{\mathrm{A}}$. This feature can be utilized in determining the value of $g_{\mathrm{A}}$ for forbidden $\beta$ transitions. This method is called the spectrum-shape method (SSM) and was introduced in. ${ }^{70}$ Further systematic studies using the SSM were performed in. ${ }^{61,71,72}$ The status of the effective values of $g_{\mathrm{A}}$ in $\beta$ and $\beta \beta$ decays is summarized in ${ }^{73}$ and the impact of the effective values of $g_{\mathrm{A}}$ on the sensitivities of the presently running and future $\beta \beta$-decay experiments has been discussed in. ${ }^{74}$ The spectrum-shape method can also be used to study the enhancement in the axial-charge matrix element (for an overwiev of the problem, see ${ }^{73}$ ) for the first-forbidden non-unique $J^{+} \leftrightarrow J^{-}$transitions.

\section{Schematic outline of the theory}

In the allowed nuclear $\beta^{-}\left(\beta^{+}\right)$transitions the emitted leptons, electron antineutrino and electron (electron neutrino and positron) carry away zero units of orbital 
angular momentum. The 0 or 1 units of change in the nuclear spin is compensated by the anti-alignment or alignment of the spins of the leptons. For the forbidden $\beta$ decays the emitted leptons carry away at least one unit of orbital angular momentum, and thus the decay amplitude is suppressed by a factor of $q R \sim 0.01$ or its higher powers. Here $R \sim$ few fm is the nuclear radius and $q \sim 1 \mathrm{MeV}$ is the momentum of the leptons. This means that the decay probability is suppressed by a huge factor (roughly by $10^{-4}$ ) each time the forbiddenness increases by one unit.

\subsection{Forbidden unique $\beta$ decays}

The forbidden unique $\beta$ transitions are the simplest ones that mediate $\beta$ decays between nuclear states of (large) angular-momentum difference $\Delta J$. In particular, if one of the states is a $0^{+}$state, then for a $K^{t h}$ forbidden $(K=1,2,3, \ldots)$ unique beta decay the angular momentum of the other involved state is $J=K+1$. At the same time the parity changes in the odd-forbidden and remains the same in the even-forbidden decays. ${ }^{75}$ The change in angular momentum and parity for different degrees of forbiddenness is presented in Table 1, and they obey the simple rule

$$
(-1)^{\Delta J} \Delta \pi=-1 . \quad \text { (Forbidden unique decays) }
$$

Table 1. Change in angular momentum and parity in $K^{t h}$ forbidden unique $\beta$ decays with a $0^{+}$state as an initial or final nuclear state.

\begin{tabular}{lrrrrrrr}
\hline $\mathrm{K}$ & 1 & 2 & 3 & 4 & 5 & 6 & 7 \\
\hline$\Delta J$ & 2 & 3 & 4 & 5 & 6 & 7 & 8 \\
$\Delta \pi=\pi_{i} \pi_{f}$ & -1 & +1 & -1 & +1 & -1 & +1 & -1 \\
\hline
\end{tabular}

The theoretical half-lives $t_{1 / 2}$ of $K^{\text {th }}$ forbidden unique $\beta$ decays can be expressed in terms of reduced transition probabilities $B_{K \mathrm{u}}$ and phase-space factors $f_{K \mathrm{u}}$. The $B_{K \mathrm{u}}$ is given by the NME, which in turn is given by the single-particle NMEs and one-body transition densities. Then (for further details see ${ }^{75}$ )

$$
t_{1 / 2}=\frac{\kappa}{f_{K \mathrm{u}} B_{K \mathrm{u}}} ; \quad B_{K \mathrm{u}}=\frac{g_{\mathrm{A}}^{2}}{2 J_{i}+1}\left|M_{K \mathrm{u}}\right|^{2},
$$

where $J_{i}$ is the angular momentum of the mother nucleus and $\kappa$ is a constant with value $^{76}$

$$
\kappa=\frac{2 \pi^{3} \hbar^{7} \ln 2}{m_{e}^{5} c^{4}\left(G_{\mathrm{F}} \cos \theta_{\mathrm{C}}\right)^{2}}=6147 \mathrm{~s},
$$

with $G_{\mathrm{F}}$ being the Fermi constant and $\theta_{\mathrm{C}}$ being the Cabibbo angle. The phase-space factor $f_{K u}$ for the $K^{t h}$ forbidden unique $\beta^{ \pm}$decay can be written as

$$
f_{K \mathrm{u}}=\left(\frac{3}{4}\right)^{K} \frac{(2 K) ! !}{(2 K+1) ! !} \int_{1}^{w_{0}} C_{K \mathrm{u}}\left(w_{e}\right) p_{e} w_{e}\left(w_{0}-w_{e}\right)^{2} F_{0}\left(Z_{f}, w_{e}\right) \mathrm{d} w_{e},
$$


where $C_{K \mathrm{u}}$ is the shape factor for $K^{\text {th }}$ forbidden unique $\beta$ decays which can be written as (see, e.g. ${ }^{75,77}$ )

$$
C_{K \mathrm{u}}\left(w_{e}\right)=\sum_{k_{e}+k_{\nu}=K+2} \frac{\lambda_{k_{e}} p_{e}^{2\left(k_{e}-1\right)}\left(w_{0}-w_{e}\right)^{2\left(k_{\nu}-1\right)}}{\left(2 k_{e}-1\right) !\left(2 k_{\nu}-1\right) !},
$$

where the indices $k_{e}$ and $k_{\nu}$ (both $k=1,2,3 \ldots$ ) come from the partial-wave expansion of the electron $(e)$ and neutrino $(\nu)$ wave functions. Here $w_{e}$ is the total energy of the emitted electron/positron, $p_{e}$ is the electron/positron momentum, $Z_{f}$ is the charge number of the daughter nucleus and $F_{0}\left(Z_{f}, w_{e}\right)$ is the Fermi function taking into account the coulombic attraction/repulsion of the electron/positron and the daughter nucleus ${ }^{\mathrm{a}}$. The factor $\lambda_{k_{e}}$ contains the generalized Fermi function $F_{k_{e}-1}{ }^{78}$ as the ratio

$$
\lambda_{k_{e}}=\frac{F_{k_{e}-1}\left(Z_{f}, w_{e}\right)}{F_{0}\left(Z_{f}, w_{e}\right)} .
$$

The integration is performed over the total (by electron rest-mass) scaled energy of the emitted electron/positron, $w_{0}$ being the endpoint energy, corresponding to the maximum electron/positron energy in a given transition.

The NME in (2) can be expressed as

$$
M_{K \mathrm{u}}=\sum_{a b} M^{(K \mathrm{u})}(a b)\left(\psi_{f}\left\|\left[c_{a}^{\dagger} \tilde{c}_{b}\right]_{K+1}\right\| \psi_{i}\right)
$$

where the factors $M^{(K \mathrm{u})}(a b)$ are the single-particle matrix elements and the quantities $\left(\psi_{f}\left\|\left[c_{a}^{\dagger} \tilde{c}_{b}\right]_{K+1}\right\| \psi_{i}\right)$ are the one-body transition densities with $\psi_{i}$ being the initial-state wave function and $\psi_{f}$ the final-state wave function. The operator $c_{a}^{\dagger}$ is a creation operator for a nucleon in an orbital $a$ and the operator $\tilde{c}_{a}$ is the corresponding annihilation operator. The single-particle matrix elements are given (in the Biedenharn-Rose phase convention) by

$$
M_{K \mathrm{u}}(a b)=\sqrt{4 \pi}\left(a\left\|r^{K}\left[Y_{K} \boldsymbol{\sigma}\right]_{K+1} i^{K}\right\| b\right),
$$

where $Y_{K}$ is a spherical harmonic of $\operatorname{rank} K, \boldsymbol{\sigma}$ a vector containing the Pauli matrices as its components, $r$ the radial coordinate, and $a$ and $b$ stand for the single-particle orbital quantum numbers. The NME (8) is given explicitly in. ${ }^{75}$

\subsection{Forbidden non-unique $\beta$ decays}

In the forbidden non-unique $\beta$ decay the half-life can be given, analogously to (2), in the form

$$
t_{1 / 2}=\kappa / \tilde{C}
$$

${ }^{\text {a } F o r ~ p o s i t r o n ~ e m i s s i o n ~ t h e ~ c h a n g e ~} Z_{f} \rightarrow-Z_{f}$ has to be performed in $F_{0}\left(Z_{f}, w_{e}\right)$ and $F_{k_{e}-1}\left(Z_{f}, w_{e}\right)$, Eq. (6) below. 
where $\tilde{C}$ is the dimensionless integrated shape function, given by

$$
\tilde{C}=\int_{1}^{w_{0}} C\left(w_{e}\right) p w_{\mathrm{e}}\left(w_{0}-w_{e}\right)^{2} F_{0}\left(Z_{f}, w_{e}\right) \mathrm{d} w_{e},
$$

with the notation explained in Sec. 2.1. The general form of the shape factor of Eq. (10) is a sum

$$
C\left(w_{e}\right)=\sum_{k_{e}, k_{\nu}, K} \lambda_{k_{e}}\left[M_{K}\left(k_{e}, k_{\nu}\right)^{2}+m_{K}\left(k_{e}, k_{\nu}\right)^{2}-\frac{2 \gamma_{k_{e}}}{k_{e} w_{e}} M_{K}\left(k_{e}, k_{\nu}\right) m_{K}\left(k_{e}, k_{\nu}\right)\right],
$$

where the factor $\lambda_{k_{e}}$ was given in (6) and $Z_{f}$ is the charge number of the final nucleus. The indices $k_{e}$ and $k_{\nu}(k=1,2,3 \ldots)$ are related to the partial-wave expansion of the electron $(e)$ and neutrino $(\nu)$ wave functions, $K$ is the order of forbiddenness of the transition, and $\gamma_{k_{e}}=\sqrt{k_{e}^{2}-\left(\alpha Z_{f}\right)^{2}}, \alpha \approx 1 / 137$ being the fine-structure constant. The nuclear-physics information is hidden in the factors $M_{K}\left(k_{e}, k_{\nu}\right)$ and $m_{K}\left(k_{e}, k_{\nu}\right)$, which are complicated combinations of different NMEs and leptonic phase-space factors. For more information on the integrated shape function, see. ${ }^{78,79}$

The shape factor $C\left(w_{e}\right)(11)$ can be decomposed into vector, axial-vector and mixed vector-axial-vector parts in the form ${ }^{70}$

$$
C\left(w_{e}\right)=g_{\mathrm{V}}^{2} C_{\mathrm{V}}\left(w_{e}\right)+g_{\mathrm{A}}^{2} C_{\mathrm{A}}\left(w_{e}\right)+g_{\mathrm{V}} g_{\mathrm{A}} C_{\mathrm{VA}}\left(w_{e}\right) .
$$

The same is true for the shape function of the forbidden unique decays (5) when the so-called next-to-leading-order terms are added to the leading ones. ${ }^{61,70}$ Integrating equation (12) over the electron kinetic energy, we obtain an analogous expression for the integrated shape function (10)

$$
\tilde{C}=g_{\mathrm{V}}^{2} \tilde{C}_{\mathrm{V}}+g_{\mathrm{A}}^{2} \tilde{C}_{\mathrm{A}}+g_{\mathrm{V}} g_{\mathrm{A}} \tilde{C}_{\mathrm{VA}},
$$

where the factors $\tilde{C}_{i}$ in Eq. (13) are just constants, independent of the electron energy.

\section{Contaminants in rare-events searches}

There is a long list of common background contaminants in dark-matter and rareevents experiments. ${ }^{80}$ Usually the $\beta$-spectrum shapes of the corresponding $\beta$ decays have not been measured or computed. Below we give few examples of the $\beta$ spectra that have also been measured, but quite long time ago (during the 50's). More such spectra will be presented elsewhere as a separate compilation.

The long-lived potassium isotope ${ }^{40} \mathrm{~K}$ is a common pollutant in the environment and in many materials. In Fig. 1 the normalized electron spectrum (the superficial area is normalized to unity) for the $\beta^{-}$decay of ${ }^{40} \mathrm{~K}$ is presented. The dominant decay channel $(89.28 \%)$ is the third-forbidden unique $\beta^{-}$decay to the ground state of ${ }^{40} \mathrm{Ca} .{ }^{81}$ The electron spectra have been computed by using the interacting shell model (ISM) with two different effective interactions: sdpfu ${ }^{82}$ and sdpfk. ${ }^{83}$ The wave functions were calculated by restricting the protons to the $s d$ shell and neutrons to 


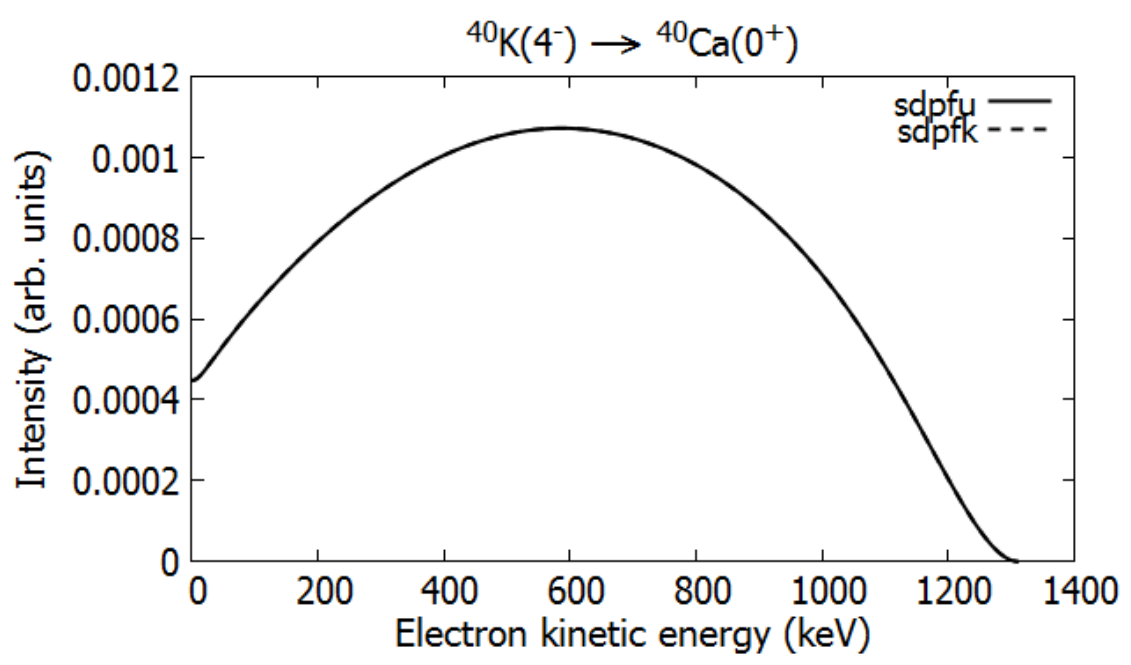

Fig. 1. Normalized $\beta$ spectrum for the third-forbidden unique ground-state-to-ground-state $\beta^{-}$ decay of ${ }^{40} \mathrm{~K}$ calculated by using two different shell-model interactions. The value $g_{\mathrm{V}}=1.00$ was adopted in the calculations.

the $s d f_{7 / 2}$ valence space, thus permitting configuration mixing for the doubly magic nucleus ${ }^{40} \mathrm{Ca}$. An old measurement of the $\beta$-spectrum shape has been reported in. ${ }^{84}$

The $\beta^{+}$decay of ${ }^{22} \mathrm{Na}$ is a common pollutant in the Ge-based experiments. ${ }^{85} \mathrm{In}$ Fig. 2 the normalized positron spectrum for the second-forbidden unique groundstate-to-ground-state $\beta^{+}$decay of ${ }^{22} \mathrm{Na}$ is depicted. The spectra have been calculated in the $s d$ valence space using the ISM with three different interactions, namely usda, usdb and usde. ${ }^{86}$ The $Q$-value of this decay is known quite accurately. ${ }^{87}$ An old measurement of the $\beta$ spectrum has been reported in..$^{88}$

The $\beta^{-}$decay of ${ }^{60} \mathrm{Co}$ is a common pollutant in the environment and in Ge-based experiments. ${ }^{85}$ In Fig. 3 the normalized electron spectra for the second-forbidden unique $\beta^{-}$decay of ${ }^{60} \mathrm{Co}$ to the first $2^{+}$state in ${ }^{60} \mathrm{Ni}$ is shown for five different values for $g_{\mathrm{A}}$. The $\beta$ spectra have been calculated by using the ISM with the Horie-Ogawa interaction. ${ }^{89,90}$ Due to the large number of valence nucleons in the $p f$ shell the calculations were truncated to the proton- $0 f_{7 / 2}$-neutron- $1 p 0 f_{5 / 2}$ subspace. Though the dominant decay channel is the allowed decay to the first $4^{+}$state in ${ }^{60} \mathrm{Ni}$ there is a small branching $(0.12 \%)$ to the first $2^{+}$state in ${ }^{60} \mathrm{Ni}^{91}$ The decomposition (12) suggests that the spectrum shape could be $g_{\mathrm{A}}$ dependent. It can be seen in the figure, however, that the next-to-leading-order corrections to the $\beta$-decay shape factor are not strong enough to make the spectrum shape $g_{\mathrm{A}}$ dependent. An old measurement of the $\beta$-spectrum shape has been reported in. ${ }^{92}$ 


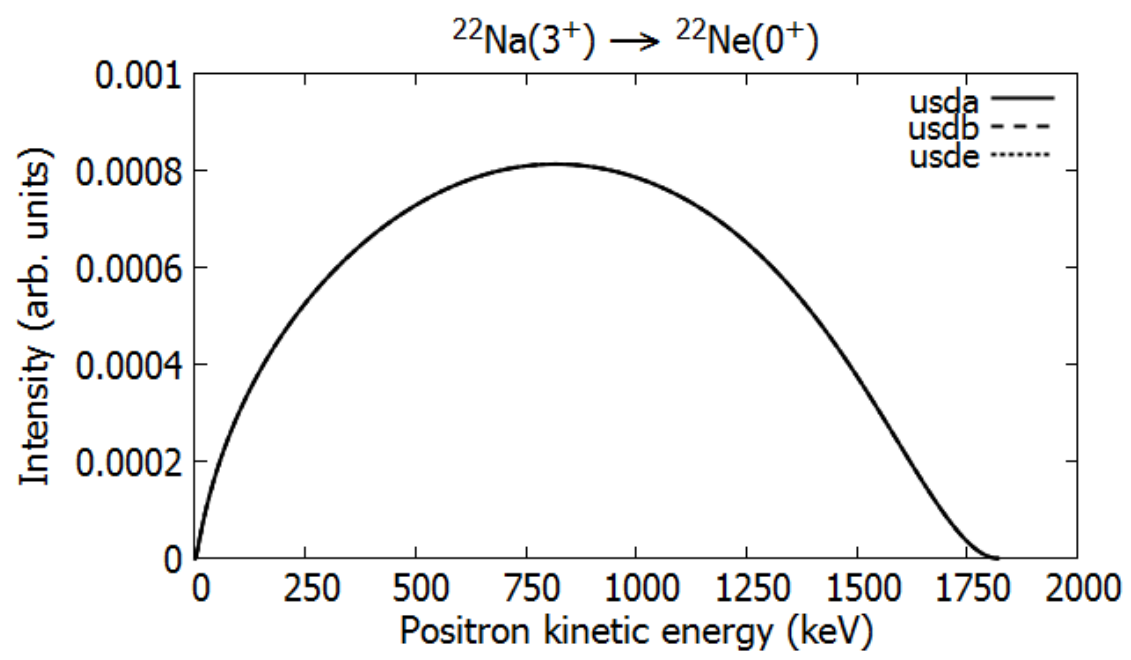

Fig. 2. Normalized $\beta$ spectrum for the second-forbidden unique ground-state-to-ground-state $\beta^{+}$ decay of ${ }^{22} \mathrm{Na}$ calculated by using three different shell-model interactions. The value $g_{\mathrm{V}}=1.00$ was adopted in the calculations.

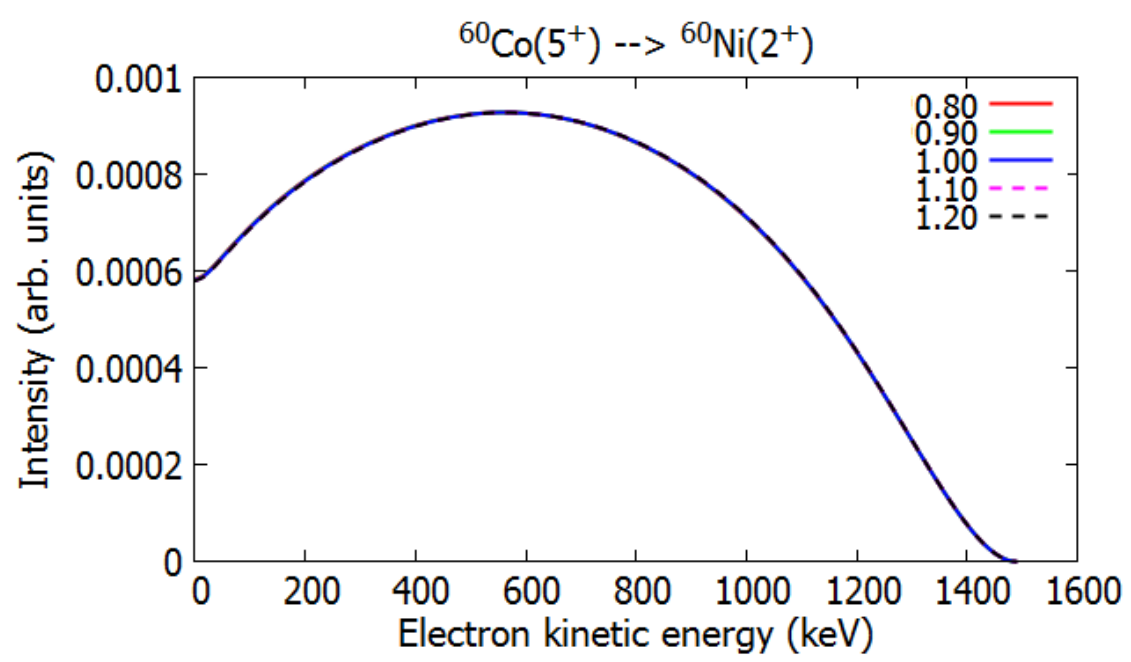

Fig. 3. Normalized $\beta$ spectra for the second-forbidden unique $\beta^{-}$decay of ${ }^{60}$ Co to the first $2^{+}$ state in ${ }^{60} \mathrm{Ni}$. The value $g_{\mathrm{V}}=1.00$ was adopted in the calculations and the color coding represents the different adopted values for $g_{\mathrm{A}}$.

\section{Reactor antineutrino anomaly}

While the actual cumulative $\beta$ spectra leading to the RAA and emerging from the decays of the fission fragments are numerous, not all of them contribute in equal 
amounts. Then the cumulative $\beta$ spectra can be nicely fit by just a quite limited number of virtual $\beta$ spectra emerging from non-existent fictional $\beta$ branches. ${ }^{57-59}$ A shortcoming of this procedure is that all the virtual branches are assumed to be described by allowed $\beta$-spectrum shapes. Also adding information from the nuclear database is not accurate enough due to deficiencies in this information. Out of the several thousand $\beta$ branches taking part in the cumulative $\beta$ spectra the majority are allowed Fermi and Gamow-Teller decays. Forbidden decays become increasingly unlikely with increasing degree of forbiddenness.

The most important $\beta$ branches taking part in the cumulative $\beta$ spectra of the RAA were identified in $^{93}$ and they include the first-forbidden decays of ${ }^{88} \mathrm{Rb}$, ${ }^{90} \mathrm{Rb},{ }^{92} \mathrm{Rb},{ }^{95} \mathrm{Sr},{ }^{96} \mathrm{Y},{ }^{100} \mathrm{Nb},{ }^{135} \mathrm{Te},{ }^{140} \mathrm{Cs}$ and ${ }^{142} \mathrm{Cs}$ from the fission of ${ }^{235} \mathrm{U}$. The branchings of these decay transitions are between $33 \%$ and $96 \%$. Here, as also in the analysis of, ${ }^{63}$ allowed $\beta$ spectrum shapes were assumed also for the forbidden transitions, like the first-forbidden decays listed above. Thus, it is of paramount importance to compute the shapes of the $\beta$ spectra associated to the above-listed key transitions and compare these spectra with the allowed shape to see the error made in the allowed approximation. The computation of the proper spectral shapes can be done by using the formalism of sections 2.1 and 2.2. An example of the application of the formalism is presented in Fig. 4 where the ISM-computed firstforbidden non-unique ground-state-to-ground-state $\beta^{-}$decay of ${ }^{140} \mathrm{Cs}$ is depicted and compared with the allowed spectrum shape. The used interaction is jj56pnb ${ }^{69}$ in the proton $3 s-2 d-1 g_{7 / 2}$ and neutron $3 p-2 f-1 h_{9 / 2}$ valence space. As can be seen there is a notable deviation from the spectrum shape of an allowed transition with the same $Q$-value. In this case there is also some dependence of the spectrum shape on the value of $g_{\mathrm{A}}$ and in other key transitions this could be the case as well, as suggested by the decomposition (12). The effects stemming from the uncertainty in the value of $g_{\mathrm{A}}$ have also been neglected in the analyses of the RAA thus far.

\section{Spectral shapes and the effective value of $g_{\mathrm{A}}$}

$\mathrm{In}^{70}$ it was found that the shapes of $\beta$ spectra could be used to determine the values of the weak coupling strengths by comparing the computed spectrum with the measured one for forbidden non-unique $\beta$ decays. This method was coined the spectrum-shape method (SSM). In this study also the next-to-leading-order corrections to the $\beta$-decay shape factor were included. $\operatorname{In}^{70}$ the $\beta$-electron spectra were studied for the 4th-forbidden non-unique ground-state-to-ground-state $\beta^{-}$decay branches ${ }^{113} \operatorname{Cd}\left(1 / 2^{+}\right) \rightarrow{ }^{113} \operatorname{In}\left(9 / 2^{+}\right)$and ${ }^{115} \operatorname{In}\left(9 / 2^{+}\right) \rightarrow{ }^{115} \operatorname{Sn}\left(1 / 2^{+}\right)$using the microscopic quasiparticle-phonon model $(\mathrm{MQPM})^{94}$ and the ISM. It was verified by both nuclear models that the $\beta$ spectrum shapes of both transitions are highly sensitive to the values of $g_{\mathrm{V}}$ and $g_{\mathrm{A}}$ and hence comparison of the calculated spectrum shape with the measured one opens a way to determine the values of these 


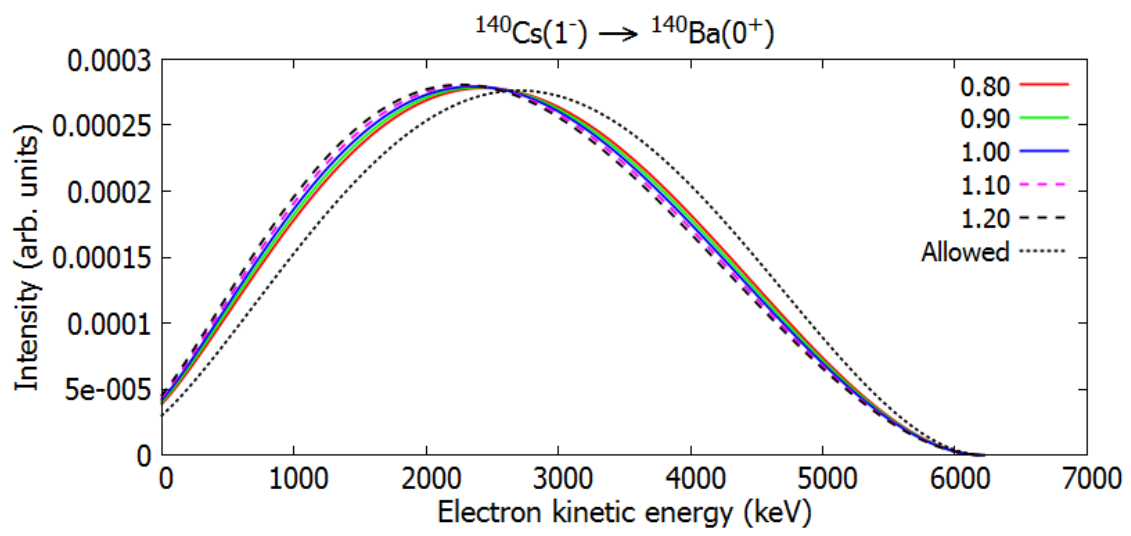

Fig. 4. Normalized $\beta$ spectrum for the first-forbidden non-unique ground-state-to-ground-state $\beta^{-}$decay of ${ }^{140} \mathrm{Cs}$. The value $g_{\mathrm{V}}=1.00$ was adopted in the calculations and the color coding represents the different adopted values for $g_{\mathrm{A}}$. The allowed spectrum shape is plotted for comparison.

coupling strengths ${ }^{\mathrm{b}}$. As a by-product it was found that for all values of $g_{\mathrm{A}}$ the best fits to data were obtained by using the canonical $\mathrm{CVC}$ value $g_{\mathrm{V}}=1.0$ for the vector coupling strength. The work of ${ }^{70}$ was extended to other nuclei and nuclear models in. ${ }^{61,70-72}$ In these studies it was found that the SSM is very robust, quite insensitive to the adopted mean field and nuclear model and its model Hamiltonian used to produce the wave functions of the participant initial and final nuclear states.

Table 2. List of forbidden non-unique $\beta^{-}$-decay transitions and their sensitivity to the value of $g_{\mathrm{A}}$. Here $J_{i}\left(J_{f}\right)$ is the angular momentum of the initial (final) state, $\pi_{i}\left(\pi_{f}\right)$ the parity of the initial (final) state, and $K$ the degree of forbiddenness. The initial state is always the ground state (gs, column 2) of the mother nucleus and the final state is either the ground state (gs) or the $n_{f}: t h, n_{f}=2,3$, excited state (column 3 ) of the daughter nucleus. The branchings to the indicated final states are practically $100 \%$ in all cases. Column 4 indicates the sensitivity to the value of $g_{\mathrm{A}}$, and the last column lists the nuclear models which have been used (thus far) to compute the $\beta$-spectrum shape. Here also references to the original works are given.

\begin{tabular}{ccccll}
\hline \multicolumn{1}{c}{ Transition } & $J_{i}^{\pi_{i}}(\mathrm{gs})$ & $J_{f}^{\pi_{f}}\left(n_{f}\right)$ & $K$ & Sensitivity & Nucl. model \\
\hline${ }^{87} \mathrm{Rb} \rightarrow{ }^{87} \mathrm{Sr}$ & $3 / 2^{-}$ & $9 / 2^{+}(\mathrm{gs})$ & 3 & Moderate & MQPM, ${ }^{71} \mathrm{ISM}^{72}$ \\
${ }^{94} \mathrm{Nb} \rightarrow{ }^{94} \mathrm{Mo}$ & $6^{+}$ & $4^{+}(2)$ & 2 & Strong & ISM $^{72}$ \\
${ }^{98} \mathrm{Tc} \rightarrow{ }^{98} \mathrm{Ru}$ & $6^{+}$ & $4^{+}(3)$ & 2 & Strong & ISM $^{72}$ \\
${ }^{99} \mathrm{Tc} \rightarrow{ }^{99} \mathrm{Ru}$ & $9 / 2^{+}$ & $5 / 2^{+}(\mathrm{gs})$ & 2 & Strong & $\mathrm{MQPM}^{71} \mathrm{ISM}^{72}$ \\
${ }^{113} \mathrm{Cd} \rightarrow{ }^{113} \mathrm{In}$ & $1 / 2^{+}$ & $9 / 2^{+}(\mathrm{gs})$ & 4 & Strong & MQPM, ${ }^{70,71} \mathrm{ISM}^{70} \mathrm{IBFM}^{61}$ \\
${ }^{115} \mathrm{In} \rightarrow{ }^{115} \mathrm{Sn}$ & $9 / 2^{+}$ & $1 / 2^{+}(\mathrm{gs})$ & 4 & Strong & MQPM, \\
${ }^{210} \mathrm{Bi} \rightarrow{ }^{210} \mathrm{Po}$ & $1^{-}$ & $0^{+}(\mathrm{gs})$ & 1 & Strong & ISM (this work) ${ }^{61} \mathrm{IBFM}-2^{61}$ \\
\hline
\end{tabular}

Examples of possible $g_{\mathrm{A}}$ dependencies are given in the previously discussed Fig. 4

${ }^{\mathrm{b}}$ This effect was overlooked in the earlier studies in Refs. ${ }^{79,95}$ 
10 Authors' Names
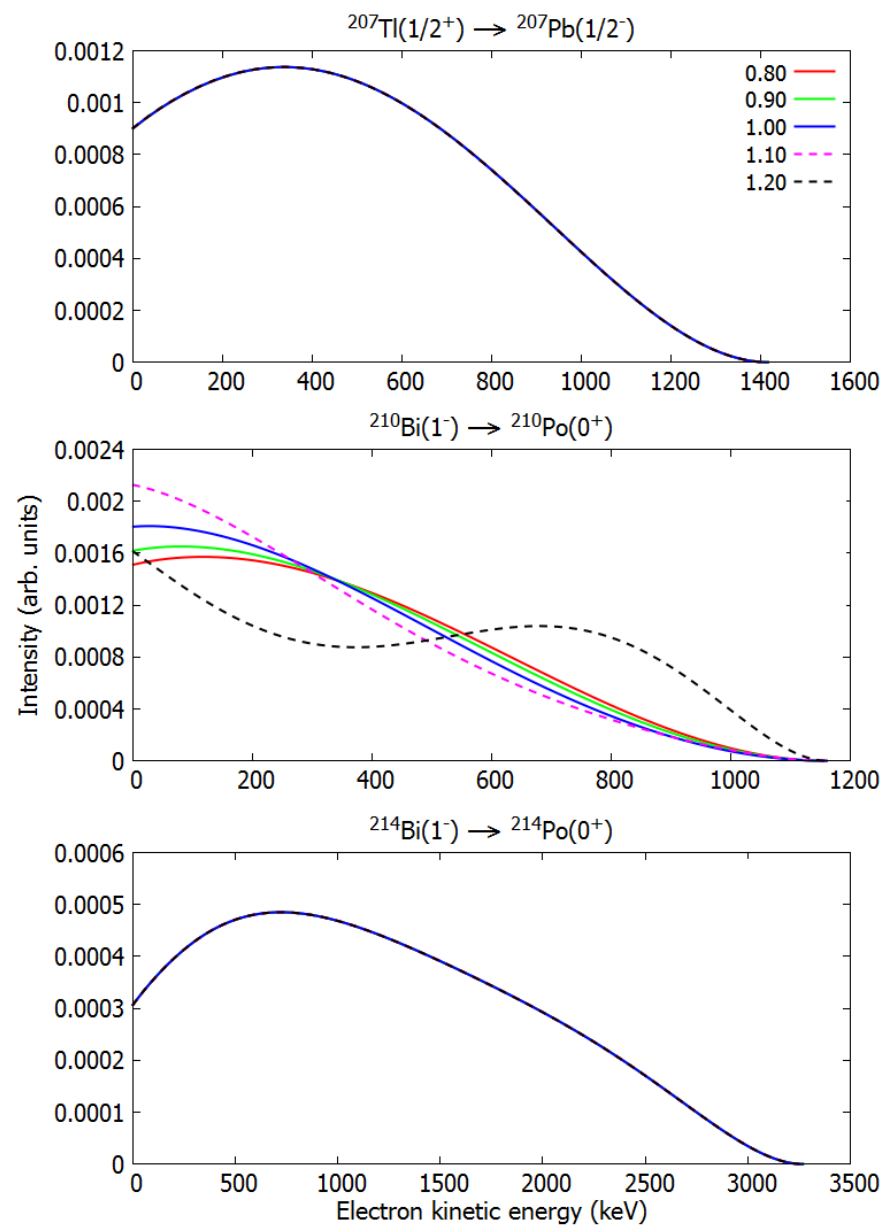

Fig. 5. Normalized $\beta$ spectra for the first-forbidden non-unique ground-state-to-ground-state $\beta^{-}$ decays of ${ }^{207} \mathrm{Tl}$ [panel (a)], ${ }^{210} \mathrm{Bi}$ [panel (b)] and ${ }^{214} \mathrm{Bi}\left[\right.$ panel (c)]. The value $g_{\mathrm{V}}=1.00$ was adopted in the calculations and the color coding represents the different adopted values for $g_{\mathrm{A}}$.

and in Fig. 5 where the ISM-computed first-forbidden non-unique ground-state-toground-state $\beta^{-}$decays of ${ }^{207} \mathrm{Tl}$ [panel (a)], ${ }^{210} \mathrm{Bi}$ [panel (b)] and ${ }^{214} \mathrm{Bi}$ [panel (c)] are depicted. The wave functions related to the decay of ${ }^{207} \mathrm{Tl}$ were calculated using the interaction $\mathrm{khhe}^{96}$ in a valence space spanned by the proton orbitals $0 g_{7 / 2}, 1 d$, $2 s$ and $0 h_{11 / 2}$, and the neutron orbitals $0 h_{9 / 2}, 1 f, 2 p$ and $0 i_{13 / 2}$. For the heavier nuclei, ${ }^{210} \mathrm{Bi}$ and ${ }^{214} \mathrm{Bi}$, the interaction khpe ${ }^{96}$ was adopted. For ${ }^{210} \mathrm{Bi}$ the valence space was spanned by the proton orbitals $0 h_{9 / 2}, 1 f, 2 p$ and $0 i_{13 / 2}$, and neutron orbitals $0 i_{11 / 2}, 1 g$ and $2 d_{5 / 2}$. For ${ }^{214} \mathrm{Bi}$ excitations to the neutron $2 d-3 s$ orbitals were not allowed to reduce the formidable computational burden.

The $\beta$-spectrum shapes of ${ }^{207} \mathrm{Tl}$ and ${ }^{214} \mathrm{Bi}$ are only slightly $g_{\mathrm{A}}$ dependent, but for 
${ }^{210} \mathrm{Bi}$ the dependence is extremely strong. This makes ${ }^{210} \mathrm{Bi}$ an excellent candidate for the application of the SSM. This is so far the only known first-forbidden $\beta$ transition with a strong $g_{\mathrm{A}}$ dependence. There is also an old $\beta$-spectrum measurement reported in. ${ }^{97}$ Other strongly $g_{\mathrm{A}}$ dependent decay transitions are listed in Table 2 . Table 2 summarizes the exploratory works of ${ }^{61,70-72}$ in terms of listing the studied $\beta$-decay transitions which are potentially measurable in rare-events experiments. Usually only the non-unique forbidden $\beta$-decay transitions can be sensitive enough to $g_{\mathrm{A}}$ to be measured even when the next-to-leading-order terms are included in the $\beta$-decay shape factor. ${ }^{70}$

\section{Enhancement of the axial-charge matrix element}

The enhancement of the axial-charge NME $\gamma_{5}$ due to nuclear medium effects in the form of meson-exchange currents was first suggested nearly four decades ago. ${ }^{98-100}$ An enhancement of 40-70\% over the impulse-approximation value was predicted based on chiral-symmetry arguments and soft-pion theorems. This enhancement seems fundamental in nature and insensitive to nuclear-structure aspects. ${ }^{101,102}$ Systematic shell-model studies of the $\gamma_{5}$ matrix elements in the $A \approx 16, A \approx 40$, and $A \approx 208$ regions indicated enhancements of $60-100 \% .{ }^{103-105} \operatorname{In}^{106}$ the exceptionally large enhancement of the $\gamma_{5}$ NME in heavy nuclei, witnessed in the shell-model studies of Warburton, ${ }^{105}$ was reproduced by introducing an effective Lagrangian incorporating approximate chiral and scale invariance of the QCD.

The $\gamma_{5}$ NME is one of the two rank-zero matrix elements contributing to firstforbidden $\Delta J=0, J^{+} \leftrightarrow J^{-}$, transitions. It plays quite an important role in the decay rates of many of these transitions. Therefore, a significant enhancement of this matrix element can also affect the shapes of the corresponding beta spectra.

For our discussion we adopt the expansion of Behrens and Bühring, ${ }^{78}$ where in the leading order the non-relativistic reduction produces two NMEs that mediate $J^{+} \leftrightarrow J^{-}$type of decay transitions, see. ${ }^{61}$ These NMEs correspond to the operators

$$
\begin{gathered}
g_{\mathrm{A}}\left(\gamma_{5}\right) \boldsymbol{\sigma} \cdot \mathbf{p}_{e}, \\
g_{\mathrm{A}} \boldsymbol{\sigma} \cdot \mathbf{r},
\end{gathered}
$$

where $\mathbf{r}$ is the radial coordinate, $\mathbf{p}_{e}$ is the electron momentum and $\boldsymbol{\sigma}$ contains the Pauli matrices. Here the enhancement of the $\gamma_{5} \operatorname{NME~}\left(\boldsymbol{\sigma} \cdot \mathbf{p}_{e}\right.$ in the non-relativistic reduction) is included in the coupling strength by

$$
g_{\mathrm{A}}\left(\gamma_{5}\right)=\left(1+\varepsilon_{\mathrm{MEC}}\right) g_{\mathrm{A}},
$$

where the enhancement $\varepsilon_{\mathrm{MEC}}$ stems from the meson-exchange currents (MEC). In this work we include also the next-to-leading-order terms in the Behrens-Bühring expansion. ${ }^{78}$ The atomic screening effects and radiative corrections are also included in the shape factor. Details of the formalism can be found in Ref. ${ }^{61}$

Our ISM calculations were performed in the following valence spaces: For the decay of ${ }^{95} \mathrm{Sr}$ a model space including the proton orbitals $0 f_{5 / 2}, 1 p_{3 / 2}, 1 p_{1 / 2}$ and $0 g_{9 / 2}$, 


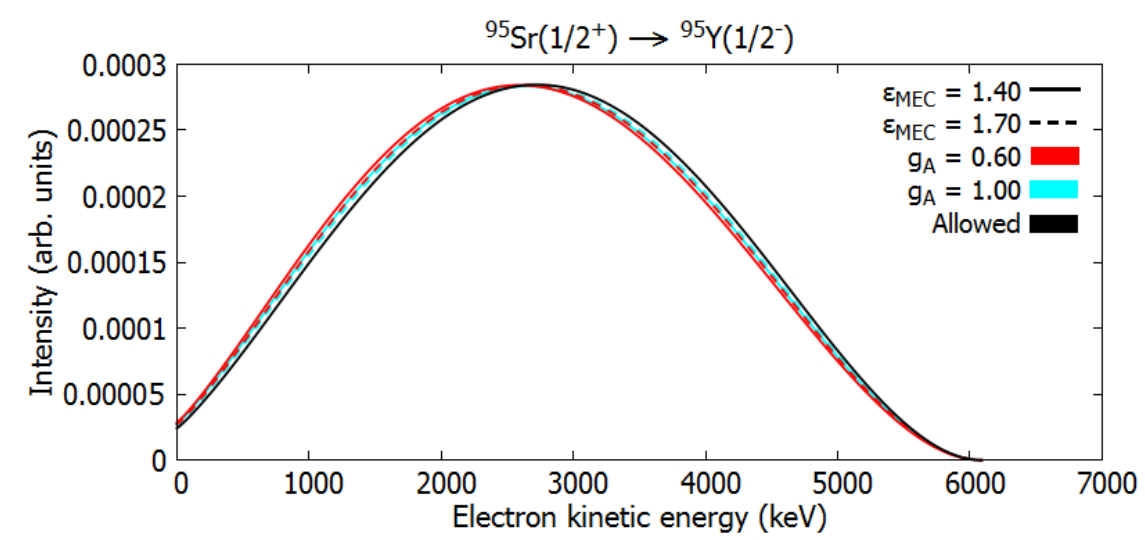

Fig. 6. Normalized $\beta$ spectra for the first-forbidden non-unique ground-state-to-ground-state $\beta^{-}$ decay of ${ }^{95} \mathrm{Sr}$. The value $g_{\mathrm{V}}=1.00$ was adopted in the calculations and the color coding represents the different adopted values for $g_{\mathrm{A}}$ and the enhancement $\left(\varepsilon_{\mathrm{MEC}}\right)$ of the axial charge.

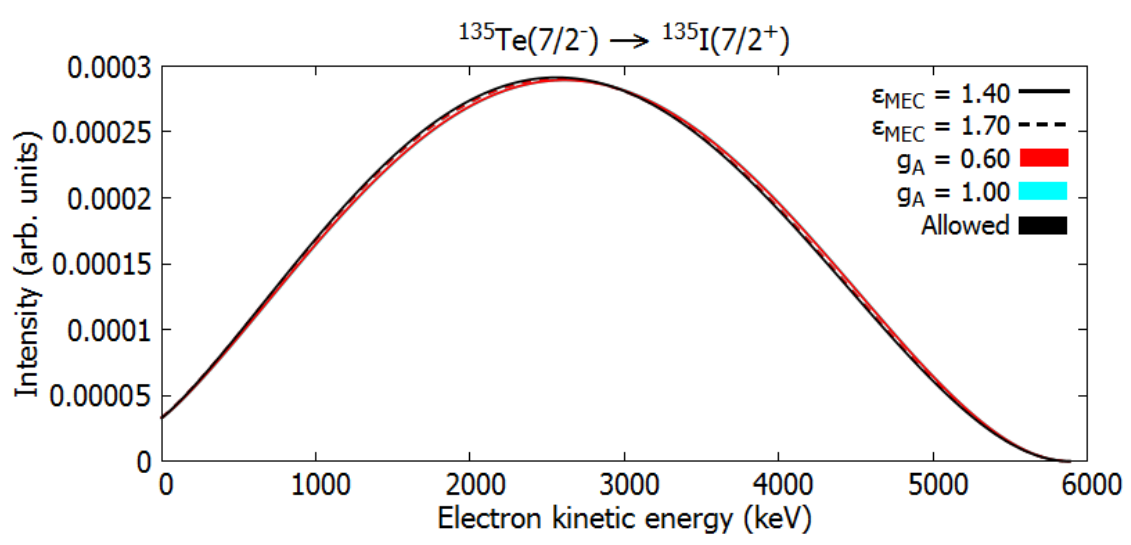

Fig. 7. Normalized $\beta$ spectra for the first-forbidden non-unique ground-state-to-ground-state $\beta^{-}$ decay of ${ }^{135} \mathrm{Te}$. The value $g_{\mathrm{V}}=1.00$ was adopted in the calculations and the color coding represents the different adopted values for $g_{\mathrm{A}}$ and the enhancement $\left(\varepsilon_{\mathrm{MEC}}\right)$ of the axial charge.

and the neutron orbitals $1 d_{5 / 2}, 1 d_{3 / 2}$ and $0 s_{1 / 2}$ was used together with the interaction glbepn. ${ }^{107}$ The interaction glbepn is a bare G-matrix interaction which also has an adjusted version glepn, where two-body matrix elements from Gloeckner ${ }^{108}$ and $\mathrm{Ji}$ and Wildenthal ${ }^{109}$ have been adopted. The decay of ${ }^{135} \mathrm{Te}$ was calculated using a model space spanned by the proton orbitals $0 g_{7 / 2}, 1 d_{5 / 2}, 1 d_{3 / 2}, 2 s_{1 / 2}$ and $0 h_{11 / 2}$, and the neutron orbitals $0 h_{9 / 2}, 1 f_{7 / 2}, 1 f_{5 / 2}, 2 p_{3 / 2}, 2 p_{1 / 2}$ and $0 i_{13 / 2}$ with the effective interactions jj56 $\mathrm{pnb}^{110}$

Examples of possible $g_{\mathrm{A}}$ and $g_{\mathrm{A}}\left(\gamma_{5}\right)$ dependencies of $\beta$ spectra are given in Figs. 6 and 7 where the ISM-computed first-forbidden non-unique ground-state-to-ground- 
state $\beta^{-}$decays of ${ }^{95} \mathrm{Sr}$ and ${ }^{135} \mathrm{Te}$ are depicted. It is seen that neither the effective value of $g_{\mathrm{A}}$ nor the enhancement (16) of $g_{\mathrm{A}}\left(\gamma_{5}\right)$ affect the spectrum shape in an easily measurable way. Hence, in these cases the comparison with the experimental half-lives is the only way to pin down the amount of enhancement (16), and its possible mass dependence, in the axial-charge NME. Only a further exploratory work could tell if there are nuclear transitions where the $\beta$ spectra are sensitive to the value of $g_{\mathrm{A}}\left(\gamma_{5}\right)$. It should also be borne in mind that the spectrum shapes of $J^{+} \leftrightarrow J^{-}$transitions play an important role in the investigations of the validity of the RAA.

\section{Conclusions}

In this article the electron-spectrum shapes are discussed for $\beta$-decay transitions that are important as contaminants in rare-events searches, as integral ingredients in the reactor antineutrino anomaly and sensitive to the effective value of the weak axial-vector coupling constant $g_{\mathrm{A}}$ and the weak axial charge. Quite little is known experimentally about $\beta$-spectrum shapes, in particular for the forbidden $\beta$ decays. This is why theoretical calculations can assist in identification of dangerous backgrounds in rare-events seaches. Allowed $\beta$-spectrum shapes are assumed in the analyses related to the reactor antineutrino anomaly and calculations of the spectrum shapes for the involved key forbidden $\beta$ decays can shed more light to the confidence level of the anomaly. In a promising new method, the spectrum-shape method, the comparison of the computed and measured $\beta$ spectra for forbidden non-unique $\beta$ decays helps in pinning down the effective value of $g_{\mathrm{A}}$. The robustness of the method is based on the observations that the computed spectra are relatively insensitive to the adopted mean-field, nuclear models and model Hamiltonians. The spectrum-shape method can also be used to pin down the amount of enhancement in the axial-charge matrix element for the first-forbidden non-unique $J^{+} \leftrightarrow J^{-}$transitions. Further exploratory theoretical work has to be done in the future and measurements of electron spectra for the potentially found key non-unique $\beta$ transitions are strongly encouraged.

\section{Acknowledgments}

This work has been partially supported by the Academy of Finland under the Finnish Centre of Excellence Programme 2012-2017 (Nuclear and Accelerator Based Programme at JYFL).

\section{References}

1. J. D. Vergados, H. Ejiri and F. Šimkovic, Int. J. Mod. Phys. E 25, 1630007 (2016).

2. J. Suhonen and O. Civitarese, Phys. Rep. 300, 123 (1998).

3. J. Suhonen and O. Civitarese, J. Phys. G: Nucl. Part. Phys. 39, 085105 (2012).

4. J. Suhonen and O. Civitarese, J. Phys. G: Nucl. Part. Phys. 39, 124005 (2012). 
5. J. Engel and J. Menéndez, Rep. Prog. Phys. 80, 046301 (2017).

6. J. Maalampi and J. Suhonen, Adv. in High Energy Phys. 2013, 505874 (2013).

7. J. Hyvärinen and J. Suhonen, Phys. Rev. C 91, 024613 (2015).

8. J. Hyvärinen and J. Suhonen, Phys. Rev. C 93, 064306 (2016).

9. AURORA Collab. (O. G. Polischuk et al.), AIP Conf. Proc. 1894, 020018 (2017).

10. GERDA Collab. (M. Agostini et al.), Nature 544, 47 (2017).

11. NEMO-3 Collab. (R. Arnold et al.), Phys. Rev. D 95, 012007 (2017).

12. COBRA Collab. (J. Ebert et al.), Phys. Rev. C 94, 024603 (2016).

13. CUORE Collab. (C. Alduino et al.), Eur. Phys. J. C 77, 532 (2017).

14. EXO-200 Collab. (J. B. Albert et al.), Nature 510, 229 (2014).

15. KamLAnD-Zen Collab. (A. Gando et al.), Phys. Rev. Lett. 117, 082503 (2016).

16. MAJORANA Collab. (V. E. Guiseppe et al.), AIP Conf. Proc. 1894, 020010 (2017).

17. SuperNEMO Collab. (D. Waters et al.), J. Phys. Conf. Series 888, 012033 (2017).

18. MOON Collab. (S. Y. Young et al.), Proc. of Science ICRC2017, 1056 (2017).

19. AMoRE Collab. (H. Park et al.), AIP Conf. Proc. 1686, 020016 (2015).

20. LUMINEU Collab. (T. B. Becker et al.), Astropart. Phys. 72, 38 (2016).

21. CUPID Collab. (G. Wang et al.), arXiv:1504.03599 [physics.ins-det] (2015).

22. SNO+ Collab. (S. Andringa et al.), Adv. High Energy Phys. 2016, 6194250 (2016).

23. NEXT-100 Collab. (J. Martin-Albo et al.), J. High Energy Phys. 1605, 159 (2016).

24. PandaX-III Collab. (X. Chen et al.), Sci. China Phys. Mech. Astron. 60, 061011 (2017).

25. M. Aunola, J. Suhonen and T. Siiskonen, Europhys. Lett. 46, 577 (1999).

26. M. Haaranen, M. Horoi and J. Suhonen, Phys. Rev. C 89, 034315 (2014).

27. H. Heiskanen, M. T. Mustonen and J. Suhonen, J. Phys. G: Nucl. Part. Phys. 34, 837 (2007)

28. M. Alanssari et al., Phys. Rev. Lett. 116, 072501 (2016).

29. J. Ellis and R. Flores, Phys. Lett. B 263, 259 (1991).

30. J. Ellis and L. Roszkowski, Phys. Lett. B 283, 252 (1992).

31. J. Ellis and R. Flores, Phys. Lett. B 300, 175 (1993).

32. V. A. Bednyakov, H. Klapdor-Kleingrothaus and S. Kovalenko, Phys. Lett. B 329, 5 (1994).

33. R. Arnowitt and P. Nath, Phys. Rev. Lett. 74, 4592 (1995).

34. R. Arnowitt and P. Nath, Phys. Rev. D 54, 2374 (1996).

35. A. Bottino et al., Phys. Lett. B 402, 113 (1997).

36. XENON10 Collab. (J. Angle et al.), Phys. Rev. Lett. 107, 051301 (2011).

37. XENOn100 Collab. (E. Aprile et al.), Phys. Rev. Lett. 107, 131302 (2011).

38. XMASS Collab. (K. Abe et al.), Phys. Lett. B 759, 272 (2016).

39. ZEPLIN Collab. (C. Ghag et al.), Astropart. Phys. 35, 76 (2011).

40. PANDAX Collab. (T. Andi et al.), Phys. Rev. D 93, 122009 (2016).

41. LUX Collab. (D. S. Akerib et al.), Phys. Rev. Lett. 116, 161301 (2016).

42. CDMS Collab. (R. Agnese et al.), Phys. Rev. D 95, 082002 (2017).

43. CoGENT Collab. (C. Aalseth et al.), Phys. Rev. Lett. 106, 131301 (2011).

44. EDELWEISS Collab. (K. Eitel et al.), Physics Procedia 61, 61 (2015).

45. DAMA Collab. (R. Bernabei et al.), Eur. Phys. J. C 73, 2648 (2013).

46. KIMS Collab. (H. S. Lee et al.), Phys. Rev. D 90, 052006 (2014).

47. PICASSO Collab. (S. Archambault et al.), Phys. Lett. B 711, 153 (2012).

48. ADMX Collab. (S. J. Asztalos et al.), Phys. Rev. Lett. 104, 141301 (2010).

49. CDEX Collab. (Q. Yue et al.), Phys. Rev. D 90, 091701(R) (2014).

50. CRESST Collab. (G. Angloher et al.), Eur. Phys. J. C 74, 3184 (2014).

51. DarkSide Collab. (P. Agnes et al.), Phys. Lett. B 743, 456 (2014). 
52. DMTPC Collab. (S. Ahlen et al.), Phys. Lett. B 695, 124 (2011).

53. DRIFT Collab. (E. Daw et al.), Astropart. Phys. 35, 397 (2012).

54. SIMPLE Collab. (M. Felizardo et al.), Phys. Rev. Lett. 108, 201302 (2012).

55. C. Buck, A. P. Collin, J. Haser and M. Lindner, Phys. Lett. B 765, 159 (2017).

56. T. A. Mueller et al., Phys. Rev. C 83, 054615 (2011).

57. K. Schreckenbach, G. Colvin, W. Gelletly and F. Von Feilitzsch, Phys. Lett. B 160, 325 (1985).

58. A. A. Hahn et al., Phys. Lett. B 218, 365 (1989).

59. N. Haag, A. Gütlein, M. Hofmann, L. Oberauer, W. Potzel and K. Schreckenbach, Phys. Rev. Lett. 112, 122501 (2014).

60. E. Ydrefors, M. T. Mustonen and J. Suhonen, Nucl. Phys. A 842, 33 (2010).

61. M. Haaranen, J. Kotila and J. Suhonen, Phys. Rev. C 95, 024327 (2017).

62. F. P. An et al., Phys. Rev. Lett. 118, 251801 (2017).

63. A. C. Hayes, J. L. Friar, G. T. Garvey, G. Jungman and G. Jonkmans, Phys. Rev. Lett. 112, 202501 (2014).

64. H. Ejiri, N. Soukouti and J. Suhonen, Phys. Lett. B 729, 27 (2014).

65. H. Ejiri and J. Suhonen, J. Phys. G: Nucl. Part. Phys. 42, 055201 (2015).

66. P. Pirinen and J. Suhonen, Phys. Rev. C 91, 054309 (2015).

67. F. F. Deppisch and J. Suhonen, Phys. Rev. C 94, 055501 (2016).

68. J. Kostensalo and J. Suhonen, Phys. Rev. C 95, 014322 (2017).

69. J. M. Allmond et al., Phys. Rev. C 90, 014322 (2014).

70. M. Haaranen, P. C. Srivastava and J. Suhonen, Phys. Rev. C 93, 034308 (2016).

71. J. Kostensalo, M. Haaranen and J. Suhonen, Phys. Rev. C 95, 044313 (2017).

72. J. Kostensalo and J. Suhonen, Phys. Rev. C 96, 024317 (2017).

73. J. Suhonen, Frontiers in Physics 5, 55 (2017).

74. J. Suhonen, Phys. Rev. C 96, 055501 (2017).

75. J. Suhonen, From Nucleons to Nucleus: Concepts of Microscopic Nuclear Theory (Springer, Berlin, 2007).

76. J. C. Hardy, I. S. Towner, V. Koslowsky, E. Hagberg and H. Schmeig, Nucl. Phys. A 509, 429 (1990).

77. N. B. Gove, M. J. Martin, Nucl. Data Tables 10, 205 (1971).

78. H. Behrens, W. Bühring, Electron Radial Wave Functions and Nuclear Beta-decay (Clarendon Press, Oxford, 1982).

79. M. T. Mustonen, M. Aunola and J. Suhonen, Phys. Rev. C 73, 054301 (2006).

80. V. I. Tretyak, AIP Conf. Proc. 1894, 020026 (2017).

81. J. Chen, Nucl. Data Sheets 140, 1 (2017).

82. F. Nowacki and A. Poves, Phys. Rev. C 79, 014310 (2009).

83. K. Kaneko, Y. Sun, T. Mizusaki and M. Hasegawa, Phys. Rev. C 83, 014320 (2011).

84. W. H. Kelly, G. B. Beard and R. A. Peters, Nucl. Phys. 11, 492 (1959).

85. W.-Z. Wei, D.-M. Mei and C. Zhang, arXiv:1706.05324 [nucl-ex] (2017).

86. B. A. Brown and W. A. Richter, Phys. Rev. C 74, 034315 (2006).

87. M. S. Basunia, Nucl. Data Sheets 127, 69 (2015).

88. B. T. Wright, Phys. Rev. 90, 159 (1953).

89. H. Horie and K. Ogawa, Prog. Theor. Phys. 46, 349 (1971).

90. H. Horie and K. Ogawa, Nucl. Phys. A 216, 407 (1973).

91. E. Browne and J. Tuli, Nucl. Data Sheets 114, 1849 (2013).

92. G. L. Keister and F. H. Schmidt, Phys. Rev. 93, 140 (1954).

93. A. A. Sonzogni, T. D. Johnson and E. A. McCutchan, Phys. Rev. C 91, 011301(R) (2015).

94. J. Toivanen and J. Suhonen, Phys. Rev. C 57, 1237 (1998). 
95. M. T. Mustonen and J. Suhonen, Phys. Lett. B 657, 38 (2007).

96. E. K. Warburton and B. A. Brown, Phys. Rev. C 43, 602 (1991).

97. H. Daniel, Nucl. Phys. 31, 293 (1962).

98. K. Kubodera, J. Delorme and M. Rho, Phys. Rev. Lett. 40, 755 (1978).

99. P. Guichon, M. Giffon, J. Joseph, R. Laverriere and C. Samour, Z. Phys. A 285, 183 (1978).

100. P. Guichon, M. Giffon and C. Samour, Phys. Lett. 74B, 15 (1978).

101. I. S. Towner and F. C. Khanna, Nucl. Phys. A 372, 331 (1981).

102. J. Delorme, Nucl. Phys. A 374, 541c (1982).

103. E. K. Warburton, I. S. Towner and B. A. Brown, Phys. Rev. C 49, 824 (1994).

104. E. K. Warburton, J. A. Becker, B. A. Brown and D. J. Millener, Annals of Physics 187, 471 (1988).

105. E. K. Warburton, Phys. Rev. C 44, 233 (1991).

106. K. Kubodera and M. Rho, Phys. Rev. Lett. 67, 3479 (1991).

107. H. Mach, E. K. Warburton, R. L. Gill, R. F. Casten, J. A. Becker, B. A. Brown and J. A. Wigner, Phys. Rev. C 41, 226 (1991).

108. D. H. Gloeckner, Nucl. Phys. A 253, 301 (1975).

109. X. Ji and B. H. Wildenthal, Phys. Rev. C 37, 1256 (1988).

110. B. A. Brown, unpublished. 